\title{
About the Method of Calculating Pressure Redistribution in the Elastic Aquifer at Pumping Water Through Flowing Wells
}

\author{
NG Aloyan* \\ National Agrarian University of Armenia \\ Received: 阱 October 27, 2018; Published: 眥 October 30, 2018 \\ *Corresponding author: NG Aloyan, Department of Hydraulics, Armenia
}

\begin{abstract}
The article considers the problem of piezometric head redistribution when water intake is performed from the elastic aquifer through flowing wells. By using dimension theory and the method of least squares, we process the simulation results got from the simulation studies of flowing wells on the constant grid mathematical model and develop approximate formula for determining the pressure drawdown at any point of the suggested aquifer. Its checkup with the data of simulation and field studies has shown a satisfactory result.
\end{abstract}

Keywords: Flow rate; Flowing well; Elasticity; Piezometric head; Drawdown

\section{Introduction}

The rate of flowing wells, as well as their head in them gradually decreases over time. Thus, for hydrological calculations there have been not accepted the Theis and Jacob's formulas derived respectively for the wells that work at constant rate or with constant pressure in the well [1]. The authors in the work [2] suggest the design formula for determining the pressure drawdown in the elastic aquifer at pumping water out through flowing wells. Though, this formula for engineering use is a kind of uncomfortable, thus, herein we suggest though approximate but comparatively simple kind of the design formula.

\section{Materials and Methods}

The mentioned work [2] suggests graph dependency between dimensionless complexes $Y=Q^{*}=\frac{Q}{k m S}$ and $X=\hat{\mathbf{o}}=\frac{a t}{r^{2}}$ (Figure 1), at the processing of the results of simulation studies of flowing wells on the grid mathematical model of hydro integrator type $[3,4]$. Herein are marked: $Q$ - well rate, $K$ - filtration coefficient of aquifer, m-its thickness, S-piezometric head drawdown at distancer from the well, $a=\frac{k m}{\mu^{*}}-$ piezo conductivity of the aquifer, $\mu^{*}$ - elastic yield coefficient.

The function curve in Figure 1 has well-expressed hyperbolic shape. Let us proxy it by the formula:

$$
Y=a x^{b}, b \prec 0
$$

$a$ and $\mathrm{b}$ are constant coefficients which we will define with the help of the method of least squares.

By taking the logarithm of the equation (1), we get

$$
\ln Y=\ln a+b \ln X
$$

According to the method of least squares, the coefficients $a$ and $b$ will have good value if the sum of squared deviations is minimal, i.e.

$$
\delta_{\min }=f(a, b)=\sum_{j=1}^{N}\left(\ln a+b \ln X_{j}-\ln Y_{j}\right)^{2}=\min ,
$$

where $\mathrm{N}$ - the number of calculated values of dimensionless complexes or number of dots put on the graph in Figure 1 Considering the terms of function extremum $\mathrm{f}(a, \mathrm{~b})$, we will get the following equations:

$$
\begin{array}{r}
\frac{\partial \delta}{\partial a}=\sum_{j=1}^{N}\left(\ln a+b \ln X_{j}-\ln Y_{j}\right)=0 \\
\frac{\partial \delta}{\partial b}=\sum_{j=1}^{N}\left(\ln a+b \ln X_{j}-\ln Y_{j}\right) \ln X_{j}=0
\end{array}
$$




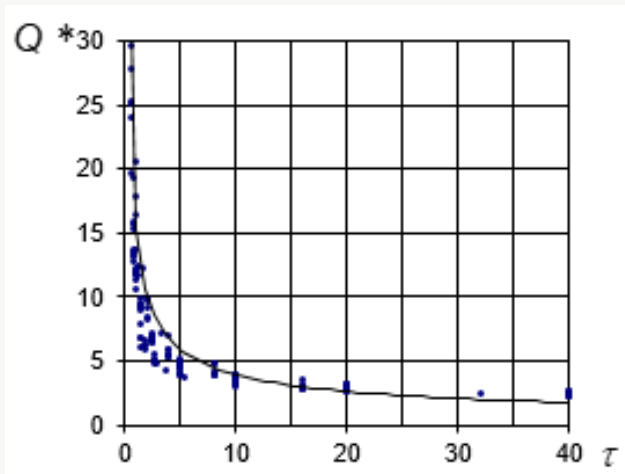

Figure 1: Graph dependency between $Q^{*}$ and $\tau$ complexes.

After elementary transformations, these equations will get the form:

$$
\begin{gathered}
N \ell n a+b \sum_{j=1}^{N} \ln X_{j}+\sum_{j=1}^{N} \ln Y_{j}=0 \\
\ln a \sum_{j=1}^{N} \ln X_{j}+b \sum_{j=1}^{N} \ln { }^{2} X_{j}-\sum_{j=1}^{N} \ell X_{j} \cdot \ln Y_{j}=0
\end{gathered}
$$

To determine the coefficient $a$, from the equation (6) we will get

$$
a=\exp \left(\frac{\sum_{j=1}^{N} \ln Y_{j}-b \sum_{j=1}^{N} \ln X_{j}}{N}\right)
$$

From the equations (7) and (8), to determine the value of coefficient $b$, we will get the following dependency:

$$
b=\frac{N \sum_{j=1}^{N} \ln X_{j} \cdot \ln Y_{j}-\sum_{j=1}^{N} \ln X_{j} \cdot \ln Y_{j}}{N \sum_{j=1}^{N} \ell n^{2} X_{j}-\left(\sum_{j=1}^{N} \ln X_{j}\right)^{2}}
$$

The values of the calculated sums of the dimensionless complexes and their products, owned from the work [5-7], are as follows:

$$
\begin{aligned}
& \sum_{j=1}^{N} \ln X_{j}=206.604 \sum_{j=1}^{N} \ln Y_{j}=301.2812 \sum_{j=1}^{N} \ln X_{j} \cdot \ln Y_{j}=222.604, \\
& \sum_{j=1}^{N} \ln ^{2} X_{j}=628.569
\end{aligned}
$$

where $\mathrm{N}=151$

By inserting these values in the equations (9) and (8), for the coefficients $\mathrm{b}$ and $a$ we will get:

$$
\begin{gathered}
b=\frac{151 \cdot 222,609-301,2812 \cdot 206,604}{151 \cdot 628,569-(206,604)^{2}}=-0,548 \\
a=\exp \left[\frac{301,2812-(-0,548) 206,604}{151}\right]=15,57 \approx 4,96 \pi
\end{gathered}
$$

Then the equation (1) will get the following form:

$$
Y=4,96 \pi X^{-0,548}
$$

\section{Conclusion}

By replacing $\mathrm{X}$ and $\mathrm{Y}$ with the values of dimensionless complexes, after some transformations, to determine the pressure drawdown at any point of the aquifer, at any time, we will get the approximate formula in its final form:

$$
S(r, t)=\frac{Q}{4,96 k m}\left(\frac{a t}{r^{2}}\right)^{0,586}
$$

The formula checkup with the data of simulation and field studies in fact has shown a satisfactory result.

\section{References}

1. Theis CV (1935) The relation between the lowering of the piezometric surface and the rate and duration of discharge of a well using groundwater storage, in: Trans. Am Geophy 16: 519-524.

2. Melikian NL, Aloyan NG, Karapetyan H (2011) Method of Hydrogeological Calculation of Flowing Wells under Steady Pressure Filtering Motion, Bulletin of Builders of Armenia 10(11): 37-44.

3. Hantusch MS (1985) Hydraulics of wells, in: Hydrology in the Service of Man. Mem.18th Congress Intern. Ass. Hydrogeol. Cambridge pp. 197206.

4. Langhaar AL (1956) Analise dimensionnels et la theoria de magusttec. Paris pp. 230.

5. Van der Kamp G (1985) Brief quantitative guidelines for the design and analysis of pumping tests. in: Hydrology in the Service of Man. Mem.18th Congress Intern. Ass. Hydrogeol Cambridge pp. 184-189.

6. Kruseman GP and Ridder NA (1994) Analysis and evaluation of pumping test data. second edition (completely revised), Netherland, pp. 377.

7. Doulkitue OA (1980) Definition of hydrogeological parameters optimal values due the work out of field data by electronic calculating machines. J Water Resources 6: 135-140. 
This work is licensed under Creative Commons Attribution 4.0 License

To Submit Your Article Click Here: Submit Article

DOI: 10.32474/CIACR.2018.05.000207

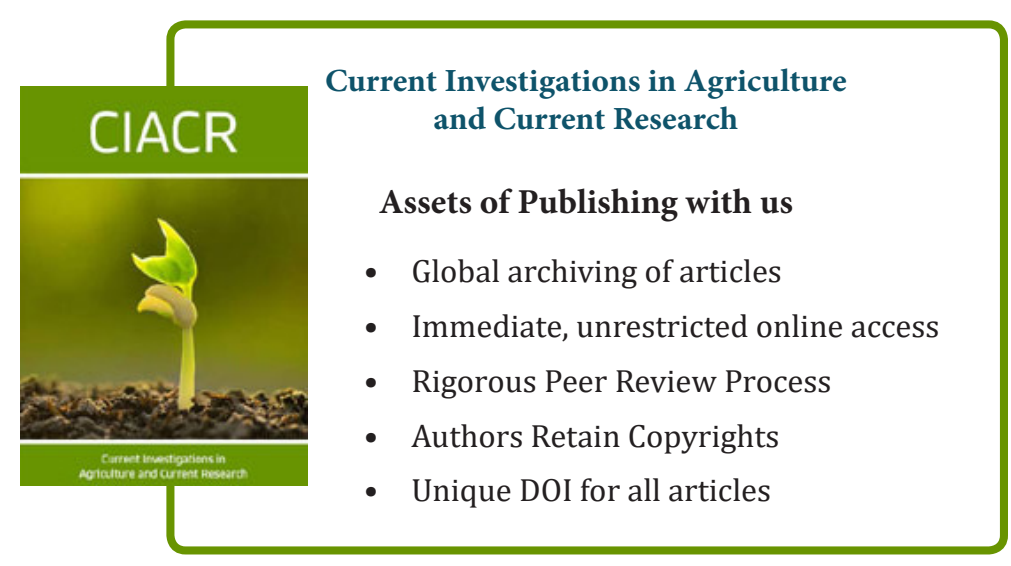

